\title{
Demographic and clinical features and rehabilitation outcomes of patients with Friedreich ataxia: A retrospective study
}

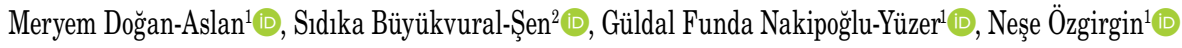 \\ ${ }^{1}$ Department of Physical Medicine and Rehabilitation, Ankara Physical Medicine and Rehabilitation Training and Research Hospital, Ankara, Turkey \\ ${ }^{2}$ Department of Physical Medicine and Rehabilitation, Çukurova Dr. Aşkım Tüfekçi State Hospital, Adana, Turkey \\ Received: October 14, 2017 Accepted: January 23, 2018 Published online: February 22, 2018
}

\begin{abstract}
Objectives: This study aims to investigate the demographic and clinical features, and rehabilitation outcomes of Friedreich Ataxia patients in the inpatient setting.

Patients and methods: Between January 2000 and December 2012, a total of 37 Friedreich Ataxia patients (26 males, 11 females; mean age $29.2 \pm 9.6$ years; range, 16 to 53 years) were included in the study. Demographic and clinical features of the patients and rehabilitation outcomes were reviewed retrospectively. Duration of rehabilitation, age at onset of disease, first onset of extremity, were determined. The Functional Ambulation Category (FAC) and Hoffer Ambulation Scale levels were used to assess the ambulation levels of patients. The Barthel Index scores were used to assess daily living activities of the patients before and after the treatment.

Results: The mean rehabilitation duration was $4.7 \pm 1.4$ weeks. There was cardiac involvement in nine (24.3\%) patients and obstructive/ restrictive pulmonary disease in five patients (13.5\%). A total of 33 patients (89.2\%) had severe ataxia, four (10.8\%) had mild ataxia, 33 (89\%) had dysarthria, 18 (48.6\%) had scoliosis, $16(43.2 \%)$ had kyphose, 15 (40.5\%) had pes cavus, five (13\%) had optic atrophy, and two (6\%) had nystagmus of all patients. At the end of the rehabilitation program, all patients were discharged with functionally improved levels based on the FAC, Hoffer Ambulation Scale $(\mathrm{p}<0.05)$, and Barthel Index $(\mathrm{p}<0.001)$, indicating a statistically significant difference.

Conclusion: An appropriate and comprehensive rehabilitation program improves the ambulation level and independence level of daily activities in patients with Friedreich ataxia, which highlights the importance of rehabilitation in these patients.
\end{abstract}

Keywords: Friedreich ataxia; inpatient rehabilitation; rehabilitation outcome.

Friedreich ataxia (FA) is a recessively inherited autosomal neurodegenerative disease, which onsets in childhood, adolescence, or early adulthood period. ${ }^{[1]}$ Respective gene was analyzed in 1996 and coded as frataxin. ${ }^{[1]}$ Frataxin has an important role on mitochondrial iron homeostasis. Frataxin deficiency causes iron and free radical accumulation in mitochondria and an increase in oxidative stress, which this results in cellular damage and death. High-energy consuming cardiac muscles, pancreas islet cells, and neural system are more sensitive to iron overload. As a result, a degeneration occurs in the dorsal root ganglion cells, posterior cord, spinocerebellar and corticospinal tract, and cerebellar dentate nucleus. ${ }^{[2]}$ The prevalence is estimated to be 3 to 4 in $100,000 .^{[3]}$ The initial symptom onset often occurs in the puberty period, and the estimated life expectancy is about 40 to 50 years. ${ }^{[3]}$ Men and women are considered to be equally affected. ${ }^{[4]}$ Ataxia, hearing loss, scoliosis, foot deformity, hypertrophic cardiomyopathy, and diabetes mellitus can be accompanied. ${ }^{[4]}$ As the nature of the disease is progressive, most of the patients become dependent on wheel chairs within 10 to 15 years. ${ }^{[3]}$ Yet, there is no pharmacological treatment, although attempts are still made to decelerate the deterioration and to develop the treatment methods. ${ }^{[4,5]}$ A majority of the cardiomyopathy patients are lost in third or fourth decade of lie due to cardiac arrhythmia or congestive failure. Kyphoscoliosis and restrictive pulmonary function disorder are also other reasons which are associated with mortality. ${ }^{[6]}$

Corresponding author: Meryem Doğan-Aslan, MD. Ankara Fizik Tedavi ve Rehabilitasyon Eğitim ve Araştırma Hastanesi Fiziksel Tip ve Rehabilitasyon Kliniği, 06230 Altındağ, Ankara, Turkey. e-mail: meryemdoganaslan@yahoo.com 
Ataxia has a progressive characteristic and initially begins from the lower extremities and spreads to the body and upper extremities. Neurological symptoms such as dysarthria, loss of deep tendon reflexes, loss of proprioception is accompanied by movement incoordination. ${ }^{[7]}$ In cranial nerve examination, decrease in visual acuity, horizontal nystagmus, hearing loss, dysarthria, and dysphagia may be present. Rapid and repeating movements are slowed down. Intentional tremor may be observed. Walking is wide-based due to the coexistence of the posterior column involvement, spasticity and cerebellar ataxia. Although asymmetrical involvement is not rare, the involvement is usually symmetrical. There is a mild dementia at the full-blown disease. Optic atrophy associated with the vision loss is not rare. ${ }^{[8]}$

Rehabilitation methods such as physical and occupational therapy, specific coordination exercises, speech therapy, posture and gait training are used in the rehabilitation of the patients with FA. ${ }^{[9]}$ Although there are a lot of genetic researches, experimental treatments and articles related with the neurological findings, orthopedic deformities and surgical interventions, cardiac pathologies for the patients with FA in the literature, there is a limited number of studies on the rehabilitation outcomes of these patients. In our study, we aimed to investigate the demographic and clinical features, functional levels, and rehabilitation outcomes of the FA patients in the inpatient setting in our hospital.

\section{PATIENTS AND METHODS}

An approval of our study was obtained from our hospital local ethics committee. A written informed consent was obtained from each patient. The study was conducted in accordance with the principles of the Declaration of Helsinki.

Our hospital is one of the largest national rehabilitation centers in Turkey. Friedreich ataxia patients, who were clinically diagnosed based on the Harding diagnostic criteria ${ }^{[10,11]}$ by a neurologist, applied to our hospital for rehabilitation. Harding diagnostic criteria were as follows: Primary: Age of onset before 25 years/autosomal recessive inheritance/progressive limb and gait ataxia/ absent tendon reflexes in the legs/extensor plantar response/axonal sensory neuropathy/dysarthria (if five years passed from onset II). Secondary: scoliosis/pyramidal weakness in lower limbs/absent reflexes in upper limbs/distal loss of position and vibration sense/cardiomyopathy III). Additional: nystagmus/optic atrophy/deafness/distal amyotrophy (weakness and wasting)/pes cavus/diabetes. However, there are significant variations about the onset, clinical findings, and the progression rate of the disease. ${ }^{[11,12]}$

A total of 37 FA patients, (26 males, 11 females; mean age $29.2 \pm 9.6$ years; range, 16 to 53 years) who received inpatient rehabilitation in our hospital between January 2000 and December 2012 years were included in the study. Archive files of patients were reviewed. Demographic and clinical features and pre- and postrehabilitation functional levels were retrospectively analyzed. Duration of rehabilitation, age (years), educational level, age at onset of disease (years), and first involvement limb of FA were determined for all patients before and after treatment.

As well as the bowels and bladder of patients, daily living activities such as feeding, bathing, grooming, transferring, mobility, toilet, and stair use were assessed with the Barthel Index. ${ }^{[13]}$ The Barthel Index is a well-documented instrument which is currently in use for measuring the outcomes of a rehabilitation and meets adequate validity and reliability. ${ }^{[14]}$ Total maximum score is 100 in this index.

The Functional Ambulation Category (FAC) ${ }^{[15,16]}$ and Hoffer Ambulation Scale ${ }^{[17,18]}$ were used to evaluate functional ambulation levels before and after treatment. The FAC has six categories ranging from 0 (non-functional ambulation) to 5 (independent). The intermediary categories include the quantify levels of assistance, supervision, and independent yet limited mobility. Ambulation levels were categorized by the Hoffer Ambulation Scale as non-ambulation, nonfunctional (therapeutic) ambulation, household, community and normal ambulation. Although the validity and reliability studies of the FAC and Hoffer Ambulation Scales for FA are not available in the literature, they are widely used scales for the rehabilitation patients and were previously used in many studies. ${ }^{[15-18]}$

None of the patients were receiving pharmacological treatment for FA. Rehabilitation of each patient was planned according to the clinical presentation of the disease. During the rehabilitation program, balance and coordination exercises, stretching and strengthening exercises, range of motion (ROM) exercises, cardiopulmonary exercises, posture exercises and ambulation training, postural biofeedback, spasticity management, occupational therapy, speech therapy, scoliosis and breathing exercises were used. A patientspecific rehabilitation program was implemented two 
hours per day during hospitalization. For ambulation, lower limb orthosis and walking aids (i.e., crutch, cane, or walker) were given to the patients who were able to ambulate functionally.

\section{Statistical analysis}

Statistical analysis was performed using the SPSS for Windows version 11.0 software (SPSS Inc., Chicago, IL, USA). Analyses were completed by transferring the data into the IBM SPSS Statistics 23.0 extension bundle (IBM Corp., Armonk, NY, USA). Categorical variables were expressed in number and percentage, while descriptive statistics were expressed in mean \pm standard deviation (SD), and median (min-max) values for the numeric variables.

For the numeric variables, the Shapiro-Wilk normalization test was used and it was observed that not all of the numeric variables were normally distributed. As numeric variables did not conform with the normal distribution, non-parametric statistical methods were used. Our FAC and Hoffer Ambulation Scale values were divided into two groups as non-ambulated/non-functionally ambulated and ambulated. The relationship between two independent categorical variables was analyzed using the chi-square test, and the relationship between two dependent categorical variables was analyzed using the McNemar's test. The differences between two dependent numeric variables were analyzed by the Wilcoxon analysis. The differences between two independent groups were investigated by the Mann-Whitney $U$ test. The correlations between two independent numeric variables were interpreted by the Spearman's Rho coefficient. A $p$ value of $<0.05$ was considered statistically significant.
The power of the study was determined via the $\mathrm{G}^{\star}$ Power 3.1 software program (Heinrich Heine University, Dusseldorf, Germany) bundle. When evaluating the power analysis, the Barthel Index results, the study's one of the most basic hypothesis, were used. Based on these results, the effect size was calculated as 0.77 . With the 0.05 significance and $95 \%$ confidence interval, the power of 37 participants was found to be $98 \%$.

\section{RESULTS}

Demographic findings including age (years), age at first symptom onset (years), rehabilitation duration (weeks) and sex (female/male), marital status (married/ single/widow), and educational level (elementary/ middle school/high school/college) of patients are shown in Table 1.

Furthermore, in all patients, the initial symptoms were ataxia and gait instability. The first involvement was the lower limb distal in 32 patients $(86.5 \%)$ and lower limb proximal in five patients (13.5\%).

Comorbidities are shown in Table 2. Cardiac involvement was the most seen systemic involvement with a rate of 9 (24.3\%). Also, 12 (32.4\%) of the patients had other systemic diseases such as obstructive/ restrictive pulmonary disease, five (13.5\%) had glomerulonephritis, hemolytic anemia, vasculitis, diabetes, and also eight (21.6\%) of the patients had a previous fracture history (upper extremity fractures $n=3$; lower extremity fractures $n=5$ ).

Urodynamic analyses were applied for the patients with neurogenic bladder in five patients (13.5\%). Three $(8.1 \%)$ of the patients were recommended using permanent urinary catheter, one $(2.7 \%)$ of

Table 1. Demographic findings of the patients with Friedreich ataxia

\begin{tabular}{|c|c|c|c|c|c|}
\hline Demographic findings & $\mathrm{n}$ & $\%$ & Mean \pm SD & Median & Min-Max \\
\hline Age (years) & & & $29.2 \pm 9.6$ & 27 & $16-53$ \\
\hline Age at first symptom onset (years) & & & $15.8 \pm 6.5$ & 14 & $5-35$ \\
\hline Rehabilitation duration (weeks) & & & $4.7 \pm 1.4$ & 4 & $2-8$ \\
\hline \multicolumn{6}{|l|}{ Gender } \\
\hline Female & 11 & 30 & & & \\
\hline Male & 26 & 70 & & & \\
\hline \multicolumn{6}{|l|}{ Marital status } \\
\hline Married & 12 & 33 & & & \\
\hline Single & 23 & 62 & & & \\
\hline Widow & 2 & 5 & & & \\
\hline \multicolumn{6}{|l|}{ Educational level } \\
\hline Elementary school & 12 & 32 & & & \\
\hline Middle school & 11 & 30 & & & \\
\hline High school & 13 & 35 & & & \\
\hline College & 1 & 3 & & & \\
\hline
\end{tabular}

SD: Standard deviation; Min: Minimum; Max: Maximum. 
Table 2. Comorbid diseases observed in our patients with Friedrich ataxia

\begin{tabular}{lcc}
\hline Comorbid diseases & $\mathrm{n}$ & $\%$ \\
\hline Cardiac problems & & \\
Minimal mitral regurgitation + tricuspid regurgitation & 2 & 5.4 \\
Minimal mitral regurgitation & 1 & 2.7 \\
Cardiomyopathy & 3 & 8.1 \\
$\quad$ Congestive cardiac failure & 3 & 8.1 \\
Visual problems & & \\
Optic atrophy & 5 & 13 \\
$\quad$ Nystagmus & 2 & 6 \\
Auditory problems & & \\
Hearing loss & 4 & 11 \\
Speech disorder & & \\
Dysarthria & 33 & 89 \\
Aphasia & 1 & 3 \\
Swallowing disorder & & \\
Dysphagia & 2 & 5.4 \\
Cognitive disorder & & \\
Cognitive dysfunction & 7 & 18.9 \\
Deformities and fracture & & \\
Pes cavus & 15 & 40.5 \\
Scoliosis & 18 & 48.6 \\
Kyphosis & 16 & 43.2 \\
Pes equinovarus & 1 & 2.7 \\
Genu recurvatum & 13 & 35.1 \\
Fracture & 8 & 21.6 \\
Mallet finger & 4 & 10.8 \\
Neurogenic bladder & & \\
Hyperreflexic synergistic bladder & 4 & 10.8 \\
Hyperreflexic dyssynergistic bladder & 1 & 2.7 \\
\hline
\end{tabular}

the patients was recommended applying a clean intermittent catheterization program, and one $(2.7 \%)$ of patients was recommended urinating spontaneously.

On neuromusculoskeletal system examination, only four (11\%) of the patients had limited ROM in their lower limbs, including the hip and ankles. Their upper extremity muscle strengths were normal, although muscle weakness was more apparent in their lower extremities. Based on the manual lower extremity muscle testing, the muscle strength was $2-3 / 5$ in 13 patients (35.1\%). Inability was observed in the cerebellar tests of 35 patients $(94.6 \%)$. Severe ataxia in 33 patients $(89.2 \%)$, mild ataxia in four patients $(10.2 \%)$, and intentional tremor in seven patients (18.9\%) were observed.

Most of the ambulated patients were able to ambulate without any assistive orthosis. Only 11 (29.7\%) of these patients were using an assistive device to ambulate. Three patients (8.1\%) were using ankle-foot orthosis (AFO), two patients (5.4\%) were using knee-ankle-foot orthosis (KAFO), and six patients $(16.2 \%)$ were using a device to prevent the knee hyperextension.

At the end of the rehabilitation program, all of the patients were discharged with functionally better levels, based on the FAC, Hoffer Ambulation Scale, and Barthel Index (Table 3). After the rehabilitation, a statistically significant difference was found in the FAC, Hoffer Ambulation Scale $(\mathrm{p}<0.05)$, and Barthel Index $(\mathrm{p}<0.001)$ (Table 4).

\section{Relationship between variables and correlation analyses}

When the correlation between age and Barthel Index values was analyzed, a negative correlation was identified $(p=0.032, r=-0.354)$. Functional level became worse with ageing. Furthermore, the mean age of the non-ambulating patients with severe ataxia

Table 3. Barthel Index scores, and Functional Ambulation Category, Hoffer Ambulation Scale scores in patients with Friedreich Ataxia at pre- and post-treatment

\begin{tabular}{|c|c|c|c|c|c|c|c|c|}
\hline \multirow[t]{2}{*}{ Functional assesment } & \multicolumn{4}{|c|}{ Pre-treatment } & \multicolumn{4}{|c|}{ Post-treatment } \\
\hline & $\mathrm{n}$ & $\%$ & Mean \pm SD & Min-Max & $\mathrm{n}$ & $\%$ & Mean \pm SD & Min-Max \\
\hline Barthel Index & & & $46.8 \pm 8.8$ & $35-65$ & & & $54.2 \pm 10.3$ & $40-70$ \\
\hline \multicolumn{9}{|c|}{ Functional Ambulation Category level } \\
\hline 0 & 11 & 29.7 & & & 10 & 20.7 & & \\
\hline 1 & 8 & 21.6 & & & 2 & 5.4 & & \\
\hline 2 & 2 & 5.4 & & & 2 & 5.4 & & \\
\hline 3 & 15 & 40.5 & & & 9 & 24.3 & & \\
\hline 4 & 1 & 2.7 & & & 13 & 35.1 & & \\
\hline 5 & 0 & 0 & & & 1 & 2.7 & & \\
\hline \multicolumn{9}{|l|}{ Hoffer Ambulation Scale level } \\
\hline Normal ambulation & 0 & 0 & & & 1 & 2.7 & & \\
\hline Community ambulation & 1 & 2.7 & & & 17 & 45.9 & & \\
\hline Household ambulation & 21 & 56.8 & & & 10 & 27 & & \\
\hline Non-functional ambulation & 15 & 40.5 & & & 9 & 24.3 & & \\
\hline Non-ambulation & 0 & 0 & & & 0 & 0 & & \\
\hline
\end{tabular}

SD: Standard deviation; Min: Minimum; Max: Maximum. 
Table 4. Comparison of Barthel Index scores, and Functional Ambulation Category, Hoffer Ambulation Scale scores in patients with Friedreich Ataxia at pre- and post-treatment

\begin{tabular}{|c|c|c|c|c|c|c|c|c|c|}
\hline \multirow[t]{2}{*}{ Rehabilitation Outcome } & \multicolumn{4}{|c|}{ Pre-treatment } & \multicolumn{4}{|c|}{ Post-treatment } & \multirow[b]{2}{*}{$p$} \\
\hline & $\mathrm{n}$ & $\%$ & Mean \pm SD & Min-Max & $\mathrm{n}$ & $\%$ & Mean \pm SD & Min-Max & \\
\hline Barthel Index & & & $46.8 \pm 8.8$ & $35-65$ & & & $54.2 \pm 10.3$ & $40-70$ & $<0.001^{\star}$ \\
\hline Functional Ambulation Category & & & & & & & & & $0.016^{*}$ \\
\hline Non-ambulated/non-functional ambulated & 21 & 56.8 & & & 14 & 37.8 & & & \\
\hline Ambulated & 16 & 43.2 & & & 23 & 62.2 & & & \\
\hline Hoffer Ambulation Scale & & & & & & & & & $0.031^{*}$ \\
\hline Non-ambulated/non-functional ambulated & 15 & 40.5 & & & 9 & 24.3 & & & \\
\hline Ambulated & 22 & 59.5 & & & 28 & 75.7 & & & \\
\hline
\end{tabular}

SD: Standard deviation; Min: Minimum; Max: Maximum; ${ }^{\star} \mathrm{p}<0.05$ statistically significant.

was significantly higher than the ambulating patients. However, no relationship between age at the first onset of symptom and ataxia levels and FAC; Hoffer Ambulation Scale levels ( $\mathrm{p}>0.05$ ), and Barthel Index scores $(\mathrm{p}=0.772, \mathrm{r}=-0.049)$ was found.

We also evaluated the relationship between gender and rehabilitation outcomes. As a result of chi-square analysis, a statistically significant relationship was found between gender and FAC before and after the treatment. In this respect, it was found that the functional levels and ambulation of the males were significantly higher than the females' $(\mathrm{p}<0.05)$. It was observed that the male patients showed better functional outcomes.

We evaluated the relationship between ambulation levels and ataxia levels of the patients. There was a significant relationship between pre- and posttreatment FAC, Hoffer Ambulation Scale levels. In patients with mild ataxia, ambulation levels also improved.

In addition, there was a statistically significant relationship between kyphosis, scoliosis, and ambulation condition of patients. Accordingly, in the non-ambulated patients, kyphosis and scoliosis rates were significantly higher than the patients who were ambulated (kyphosis $\mathrm{p}=0.018$ scoliosis $\mathrm{p}=0.009$ ). In the patients with kyphosis and scoliosis, functional levels were found to be worse.

However, no significant relationship was found between pes cavus and functional improvement $(p>0.05)$. Also, no significant relationship was found between the rehabilitation duration $(p>0.05)$, cardiac involvement ( $\mathrm{p}>0.05)$, and functional level.

\section{DISCUSSION}

Although there are many genetic researches, experimental treatments, and articles related with neurological findings, orthopedic deformities and surgical interventions, cardiac pathologies for the patients with FA in the literature, there is a limited number of studies on the rehabilitation outcomes of these patients. Therefore, we are unable to find enough literature data to discuss our outcomes. As far as we researched, our study is one of the first researches, which presents the comprehensive rehabilitation outcomes of the patients with FA.

Reetz et al. $^{[19]}$ found that the mean age of the patients was $33.9 \pm 13.8$ years. They reported that the first onset age of the disease was $15.7 \pm 10.4$ years. The mean age of the patients in the study of Dulgeroglu et al $^{[6]}$ was $23.1 \pm 9.2$ years. In the study of Stephenson et al., ${ }^{[20]}$ the authors found that the mean age was $29.4 \pm 9.0$ years, and first onset age of the disease was 19.5 \pm 8.1 years. In the Ciancarelli et al.'s ${ }^{[21]}$ study, the mean age of the patients was $32.5 \pm 10.8$ years. In our study, the mean age was $29.2 \pm 9.6$ years, and first onset age of the disease was $15.8 \pm 6.5$ years, consistent with previous studies.

Reetz et al. ${ }^{[19]}$ assessed 592 patients in the European FA Consortium and, of these patients, 54\% females and $46 \%$ were males. There were $33.4 \%$ female and $66.7 \%$ male patients in the study of Ciancarelli et al. ${ }^{[21]}$ There were $41.4 \%$ female and $58.6 \%$ male patients in the study of Milne et al. ${ }^{[22]}$ In our study, there were $30 \%$ female and $70 \%$ male patients. In the study of Dulgeroglu et al., ${ }^{[6]}$ there were $30 \%$ female and $70 \%$ male patients. Although our rates are consistent with the other studies conducted in Turkey, this is not a prevalence study. In the literature, it has been stated that the female/male ratio is close to the each other. ${ }^{[8]}$

In the Reetz et al.'s ${ }^{[19]}$ study, it was shown that 354 $(60 \%)$ of the patients were early-onset ( $\leq 14$ years), 137 (23\%) of the patients were mid-onset (15-24 years old), and $100(17 \%)$ of the patients were late-onset $(\geq 25$ years). Followed by the mid-onset patients, early-onset 
patients had a more progressive disease, while the disease progress was slower in late-onset patients. Among our patients, the number of patients with late-onset was very low. In addition, our study is not a progression study. However, when the current results were examined, we found no relationship between the age at first onset of symptom and FAC, Hoffer Ambulation Scale levels ( $\mathrm{p}>0.05)$, and Barthel Index scores $(p=0.772, r=-0.049)$, showing the functional levels.

Dulgeroglu et al. ${ }^{[6]}$ found cardiac problems in four patients (13.3\%); Reetz et al. ${ }^{[19]}$ found cardiomyopathy in 27 patients (5\%). ${ }^{[19]}$ In our study, we found cardiac problems in nine patients $(24.3 \%)$. We believe that this is caused by early referral of the patients to the physicians for their cardiac complaints, and it may be the result of the developments of early diagnostic methods.

Pandolfo $^{[4]}$ reported that it could be observed optic atrophy in $30 \%$ of patients and sensorineural hearing loss in $20 \%$ of patients. We also observed optic atrophy in $13 \%$ and hearing loss in $11 \%$ of our patients. Accompanying visual and auditory diseases were lower in our patients.

In the Cochrane databases of Kearney et al., ${ }^{[23]}$ they found that the mean rate of incidence of pes cavus was $10 \%$. Dulgeroglu et al. ${ }^{[6]}$ found dysarthria in $56.6 \%$, nystagmus in $33.3 \%$, scoliosis in $56.6 \%$, and pes cavus in $56.6 \%$ of patients. Reetz et al. ${ }^{[19]}$ found scoliosis in $25 \%$ of patients. In our study, we found dysarthria in $89 \%$, nystagmus in $6 \%$, pes cavus in $40.5 \%$, and scoliosis in $48.6 \%$ of patients. For the patients with scoliosis, we were unable to reach enough data with regard to the scoliosis type and the angle; however, we found that orthosis or surgery was recommended to none of the patients In the light of these data, it was assumed that their scoliosis angles were small.

Goulipian et al. ${ }^{[24]}$ demonstrated that rehabilitation and orthopedic shoes reduced the pain, fatigue, falling, and ankle sprain and improved the quality of life and ability to be independent. During the rehabilitation, they reduced the ankle problems and need to use walking aids of a male patient and increased the step length, walking speed and cadence. In our study, we also improved our FA patients' functional independence by giving them an AFO or a KAFO in addition to the orthopedic shoes, and reduced the joint problems.

In another study, Stephenson et al. ${ }^{[20]}$ compared eight FA patients with eight healthy individuals. There were six male and two female patients. They found that the walking speeds of FA patients were significantly slower than the control group. They also found a negative correlation between the first onset FA age and walking parameters. They concluded that the lack of stability contributed to the gait disorders in adult FA patients. In addition, Reetz et al. ${ }^{[19]}$ found the gait instability as $78 \%$. We also found a significant relationship between ataxia level, showing the lack of the stability and FAC and Hoffer Ambulation Scale levels $(\mathrm{p}<0.05)$. Ambulation became worse in patients with severe ataxia. In this respect, our results are similar with the results of Stephenson et al. ${ }^{[20]}$ However, we observed no relationship between the age at onset of first symptom and FAC, Hoffer Ambulation Scale levels ( $p>0.05)$, and Barthel Index scores $(\mathrm{p}=0.772 \mathrm{r}=-0.049)$.

In the study of Ciancarelli et al. ${ }^{[2]}$ study which was conducted in 24 FA patients, they reported that there were also cognitive function and mood disorders. They demonstrated that the rehabilitation should be added to the conventional neuromotor approaches. As it is a chronic disease, we also believe that FA patients should receive cognitive and psychosocial rehabilitation.

For the level 1, Dulgeroglu et al. ${ }^{[6]}$ reported that functional community ambulation rate of the patients increased to $14(46.6 \%)$ at discharge, while it was $11(36.6 \%)$ at admission based on the Hoffer Ambulation Scale levels. For the level 2, they showed that the rate of patients who were household ambulation increased to $7(23.3 \%)$ at discharge, while it was $4(1.3 \%)$ at admission. In our study, according to the Hoffer Ambulation Scale levels, the rate of the functional community ambulation of the patients increased to 17 (45.9\%) at discharge, while it was $1(2.7 \%)$ at admission. The rate of the patients who were ambulating at household, decreased to $10(27 \%)$ at discharge, while it was $21(56.8 \%)$ at admission. Non-functional ambulation rate of the patients decreased from 15 (40.5\%) to $9(24.3 \%)$. Most of our patients improved to a functional community ambulation level (Table 3 ). Developments in rehabilitation methods and patient awareness about early starting of the rehabilitation program can be thought as the reasons.

Dulgeroglu et al. ${ }^{[6]}$ found a significant relation between rehabilitation duration and functional outcomes. However, we were unable to find any significant correlation in our study $(\mathrm{p}=0.0361 \mathrm{r}=-0.155)$. Dulgeroglu et al. ${ }^{[6]}$ reported that the mean rehabilitation duration was 37 days. Our mean rehabilitation duration was 4.72 weeks (average 33 days). Rehabilitation 
durations of both studies are similar. Thus, we believe that there is a need for more comprehensive studies, which would determine the optimum duration, and intensity and content of the rehabilitation program for the patients with FA.

Although Dulgeroglu et al. ${ }^{[6]}$ did not find any significant difference among the patients whose ambulation levels changing in respect of kyphoscoliosis. We found a statistically significant relationship between kyphosis, scoliosis, and the ambulation state of the patients. Accordingly, kyphosis and scoliosis rates in the non-ambulating patients were significantly higher than kyphosis and scoliosis rates in the patients who were ambulating (kyphosis $\mathrm{p}=0.018$ scoliosis $\mathrm{p}=0.009$ ). In the patients with kyphosis and scoliosis, the functional levels were worse. We believe that the ambulation would be more difficult in case of vertebral orthopedic deformities, as kyphoscoliosis and the other deformities may disturb the biomechanical and postural balance. Also, kyphoscoliosis may compress the spinal cord or radicular nerve and it may cause neuromuscular defects.

Croarkin et al. ${ }^{[12]}$ investigated the correlation between unsupported independent walking and supported walking, by using walking parameters and FA Rating Scale scores in a study, which were conducted over 14 children and 24 adolescent. The mean age was $13 \pm 2.3$ years, and the mean first onset age of the disease was $8 \pm 3.1$ years. They found that 15 patients (39.5\%) were walking with an assistance, while 23 patients (60.5\%) were able to ambulate without any support or assistance. They found no significant difference between the independent groups who were walking without any support and the group who were walking with an assistance with regard to the age and first onset age of the disease. Considering the age and ataxia levels, we found that the ataxia level was more serious in non-ambulating patients and ataxia level became worse with ageing. However, we found no relationship between the age at onset of first symptom of the disease and ataxia levels $(\mathrm{p}>0.05)$.

Croarkin et al. ${ }^{[12]}$ found a significant correlation between the FA Rating Scale score and locomotor state. They reported that the FA Rating Scale scores could be a useful tool for determining the patients who would be able to walk without any support. We found that there was a significant relationship between pre- and post-treatment FAC, Hoffer Ambulation Scale levels $(p<0.05)$. In this respect, the rate of the unsupported ambulation significantly increased in the patients with mild ataxia after the treatment. We agree with the Croarkin et al.' $\mathrm{s}^{[12]}$ opinion that ataxia evaluation is a useful method for identifying patients who would able to walk without a support.

In a retrospective study including 29 patients with FA, Milne et al. ${ }^{[22]}$ evaluated the effects of receiving inpatient rehabilitation program for independence. To evaluate the efficiency of inpatient rehabilitation, they used the Functional Independent Measurement (FIM). They also mentioned that the effects of the rehabilitation on the quality of life and disease severity should be investigated. They demonstrated that the inpatient rehabilitation was extremely important, as it increased the independence in the daily living activities and provided patients functional improvement. We evaluated the daily activities of the patients by using the Barthel Index. After the rehabilitation, we found a highly significant improvement in the Barthel Index $(\mathrm{p}<0.001)$.

The study of Milne et al. ${ }^{[22]}$ is the only study that we can discuss the exercise methods, which are implemented during the inpatient rehabilitation program. In the literature, we are unable to find any studies, which evaluate the rehabilitation outcomes of the patients undergoing inpatient rehabilitation for FA, and our results are similar with the study results of Milne et al. ${ }^{[22]}$ Similar to our treatments, Milne et al., ${ }^{[22]}$ implemented the patient-specific exercises such as stretching, balance retraining, pelvic and spinal control, spasticity management, hand function and cardiopulmonary exercises, communication and swallowing exercises and specialized equipment prescription for the rehabilitation of the 29 FA patients. Unlike our rehabilitation practices, they implemented aquatic physiotherapy and the most importantly they provided safety and risk management services to the patients with FA and developed home and community programs. Unfortunately, we cannot provide sufficient community rehabilitation services under the circumstances of Turkey. This service is critical for the patients' independent participation in social life. Unfortunately, this is the lack of our society and we believe that the social rehabilitation would be better in our country in the future.

In addition, Maring et al. ${ }^{[2]}$ reported that the main goal of physical therapists should be to increase the individual participation, independent from the environmental events as much as possible by taking into account the progressive nature of the disease. We share the same opinion, and we found that the community ambulation levels increased after the rehabilitation. 


\section{Study limitation}

Study limitation was that the selection of the inpatient rehabilitation participants. Only patients with increased awareness were admitted to the rehabilitation hospital and frequency of FA was, therefore, low. In the future studies, it should be planned to include more patients. In addition, our study is a retrospective study. Furthermore, to assess the rehabilitation outcomes, randomized-controlled, prospective studies should be planned. We believe that there is a need for more comprehensive studies, which would determine the optimum duration, intensity and content of the rehabilitation program in patients with FA.

In conclusion, there is no pharmacological treatment for slowing down the progression of FA. There is a need for comprehensive researches with regard to the content, intensity, and duration of rehabilitation program to be implemented and with respect to the sustained recovery after inpatient rehabilitation. However, as we demonstrated in our study, a convenient and comprehensive rehabilitation program increases the ambulation level and independence in daily activities of the patients. Therefore, for the patients with FA, inpatient rehabilitation is extremely important.

\section{Declaration of conflicting interests}

The authors declared no conflicts of interest with respect to the authorship and/or publication of this article.

\section{Funding}

The authors received no financial support for the research and/or authorship of this article.

\section{REFERENCES}

1. Brice A. Friedreich ataxia. Orphanet Encyclopedia. October 2004. Available from: http://www.orpha.net/data/patho/ GB/uk-friedreich.pdf

2. Maring JR, Croarkin E. Presentation and progression of Friedreich ataxia and implications for physical therapist examination. Phys Ther 2007;87:1687-96.

3. Schulz JB, Boesch S, Bürk K, Dürr A, Giunti P, Mariotti $C$, et al. Diagnosis and treatment of Friedreich ataxia: a European perspective. Nat Rev Neurol 2009;5:222-34.

4. Pandolfo M. Friedreich ataxia: the clinical picture. J Neurol 2009;256:3-8.

5. Maurel N, Diop A, Gouelle A, Alberti C, Husson I. Assessment of upper limb function in young Friedreich ataxia patients compared to control subjects using a new three-dimensional kinematic protocol. Clin Biomech (Bristol, Avon) 2013;28:386-94.

6. Dulgeroglu D, Kurtaran A, Erkin G, Kaya K, Aybay C. General properties of patients with friedreich's ataxia and rehabilitation outcomes: A retrospective study. Turk J Phys Med Rehab 2003;49:27-31.
7. Gündüz A, Apaydın H. Progressive ataxias: Clinical features and differentiel diagnosis. J Parkinson Dis Mov Disord 2007;10:72.

8. Gilroy J. Basic Neurology: Degenerative diseases. 3rd ed. New York: McGraw Hill; 2000.

9. Dombovy ML, Pippin BA. Rehabilitation concerns in degenerative movement disorders the central nevous system: In: Braddom RL, Buschbacher RM, editors. Phys Med and Rehab. 2nd ed. Philadelphia: WB Saunders Company; 1996. p. 1164-76.

10. Harding AE. Friedreich's ataxia: a clinical and genetic study of 90 families with an analysis of early diagnostic criteria and intrafamilial clustering of clinical features. Brain 1981;104:589-620.

11. Santos R, Lefevre S, Sliwa D, Seguin A, Camadro JM, Lesuisse E. Friedreich ataxia: molecular mechanisms, redox considerations, and therapeutic opportunities. Antioxid Redox Signal 2010;13:651-90.

12. Croarkin E, Maring J, Pfalzer L, Harris-Love M, Siegel K, DiProspero N. Characterizing gait, locomotor status, and disease severity in children and adolescents with Friedreich ataxia. J Neurol Phys Ther 2009;33:144-9.

13. Barthel Mahoney FL, Barthel DW. Functional Evaluation: Barthel Index. MD State Med J 1965;14:61-5.

14. Granger CV, Kelly-Heyes M, Johnston M, Deutch A, Braun S, Fiedler RC. Quality and outcome measures for medical rehabilitations. In: Braddom RL, Buschbacher RM, editors. Phys Med and Rehab. 2nd ed. Philadelphia: WB Saunders Company; 1996. p. 151-64.

15. Mehrholz J, Wagner K, Rutte K, Meissner D, Pohl M. Predictive validity and responsiveness of the functional ambulation category in hemiparetic patients after stroke. Arch Phys Med Rehabil 2007;88:1314-9.

16. Viosca E, Martínez JL, Almagro PL, Gracia A, González C. Proposal and validation of a new functional ambulation classification scale for clinical use. Arch Phys Med Rehabil 2005;86:1234-8.

17. Schoenmakers MA, Gulmans VA, Gooskens RH, Pruijs JE, Helders PJ. Spinal fusion in children with spina bifida: influence on ambulation level and functional abilities. Eur Spine J 2005;14:415-22.

18. Beauchamp M, Labelle H, Duhaime M, Joncas J. Natural history of muscle weakness in Friedreich's Ataxia and its relation to loss of ambulation. Clin Orthop Relat Res 1995;311:270-5.

19. Reetz K, Dogan I, Costa AS, Dafotakis M, Fedosov K, Giunti P, et al. Biological and clinical characteristics of the European Friedreich's Ataxia Consortium for Translational Studies (EFACTS) cohort: a cross-sectional analysis of baseline data. Lancet Neurol 2015;14:174-82.

20. Stephenson J, Zesiewicz T, Gooch C, Wecker L, Sullivan K, Jahan I, et al. Gait and balance in adults with Friedreich's ataxia. Gait Posture 2015;41:603-7.

21. Ciancarelli I, Cofini V, Carolei A. Evaluation of neuropsychological functions in patients with Friedreich ataxia before and after cognitive therapy. Funct Neurol 2010;25:81-5. 
22. Milne SC, Campagna EJ, Corben LA, Delatycki MB, Teo $\mathrm{K}$, Churchyard AJ, et al. Retrospective study of the effects of inpatient rehabilitation on improving and maintaining functional independence in people with Friedreich ataxia. Arch Phys Med Rehabil 2012;93:1860-3.

23. Kearney M, Orrell RW, Fahey M, Pandolfo M. Antioxidants and other pharmacological treatments for Friedreich ataxia. Cochrane Database Syst Rev 2012;4:CD007791.

24. Goulipian C, Bensoussan L, Viton JM, Milhe-De BV, Ramon J, Delarque A. Orthopedic shoes improve gait in Friedreich's ataxia: a clinical and quantified case study. Eur J Phys Rehabil Med 2008;44:93-8. 